\title{
Cytoprotective effects of nitrite during in vivo ischemia-reperfusion of the heart and liver
}

\author{
Mark R. Duranski, ${ }^{1}$ James J.M. Greer, ${ }^{1}$ Andre Dejam, ${ }^{2}$ Sathya Jaganmohan, ${ }^{1}$ Neil Hogg, ${ }^{3}$ \\ William Langston, ${ }^{4}$ Rakesh P. Patel, ${ }^{5}$ Shaw-Fang Yet, ${ }^{6}$ Xunde Wang, 7,8 Christopher G. Kevil, ${ }^{4}$ \\ Mark T. Gladwin, ${ }^{7,8}$ and David J. Lefer ${ }^{1}$
}

\begin{abstract}
1'Departments of Physiology and Cardiology, Louisiana State University Health Sciences Center, Shreveport, Louisiana, USA. 'Laboratory of Chemical Biology, National Institute of Diabetes and Digestive and Kidney Diseases, NIH, Bethesda, Maryland, USA. ${ }^{3}$ Department of Biophysics

and Free Radical Research Center, Medical College of Wisconsin, Milwaukee, Wisconsin, USA. ${ }^{4}$ Department of Pathology, Louisiana State University

Health Sciences Center, Shreveport, Louisiana, USA. ${ }^{5}$ Department of Pathology and Center for Free Radical Biology, University of Alabama at Birmingham, Birmingham, Alabama, USA. ${ }^{6}$ Pulmonary and Critical Care Division, Brigham and Women’s Hospital, Boston, Massachusetts, USA. ${ }^{7}$ Vascular Therapeutics Section, Cardiovascular Branch, National Heart, Lung and Blood Institute, NIH, Bethesda, Maryland, USA.
\end{abstract} ${ }^{8}$ Critical Care Medicine Department, Clinical Center, NIH, Bethesda, Maryland, USA.

\begin{abstract}
Nitrite represents a circulating and tissue storage form of NO whose bioactivation is mediated by the enzymatic action of xanthine oxidoreductase, nonenzymatic disproportionation, and reduction by deoxyhemoglobin, myoglobin, and tissue heme proteins. Because the rate of NO generation from nitrite is linearly dependent on reductions in oxygen and $\mathrm{pH}$ levels, we hypothesized that nitrite would be reduced to $\mathrm{NO}$ in ischemic tissue and exert NO-dependent protective effects. Solutions of sodium nitrite were administered in the setting of hepatic and cardiac ischemia-reperfusion (I/R) injury in mice. In hepatic $I / R$, nitrite exerted profound dose-dependent protective effects on cellular necrosis and apoptosis, with highly significant protective effects observed at near-physiological nitrite concentrations. In myocardial I/R injury, nitrite reduced cardiac infarct size by $67 \%$. Consistent with hypoxia-dependent nitrite bioactivation, nitrite was reduced to NO, S-nitrosothiols, $\mathrm{N}$-nitrosamines, and iron-nitrosylated heme proteins within 1-30 minutes of reperfusion. Nitrite-mediated protection of both the liver and the heart was dependent on NO generation and independent of eNOS and heme oxygenase-1 enzyme activities. These results suggest that nitrite is a biological storage reserve of NO subserving a critical function in tissue protection from ischemic injury. These studies reveal an unexpected and novel therapy for diseases such as myocardial infarction, organ preservation and transplantation, and shock states.
\end{abstract}

\section{Introduction}

The anion nitrite $\left(\mathrm{NO}_{2}{ }^{-}\right)$forms as a consequence of $\mathrm{NO}$ oxidation and is present at concentrations of $0.3-1.0 \mu \mathrm{M}$ in plasma and $1-20 \mu \mathrm{M}$ in tissue (1-5). This nitrite may be reduced to NO during hypoxia and acidosis $(1,4-13)$. At very low tissue $\mathrm{pH}$ and oxygen tension, nitrite may be reduced to NO by disproportionation (acidic reduction) (13) or by the enzymatic action of xanthine oxidoreductase $(6,7,12,13)$. However, within physiological ranges of $\mathrm{pH}$ and oxygen tension, nitrite has been considered an inert metabolic end product of $\mathrm{NO}$ oxidation with limited intrinsic biological activity (14). Challenging this dogma, we have recently demonstrated that near-physiological levels of nitrite are reduced to NO by reaction with deoxyhemoglobin along the physiological oxygen gradient, a chemical reaction whose rate is oxygen and $\mathrm{pH}$ dependent and that potentially contributes to hypoxic vasodilation (8). We therefore hypothesized that hypoxia-dependent NO production from nitrite in ischemic tissue might limit ischemiareperfusion (I/R) injury.

Nonstandard abbreviations used: AAR, area at risk; ALT, alanine aminotransferase; AST, aspartate aminotransferase; $\mathrm{C}_{\mathrm{T}}$, comparative threshold; HO-1, heme oxygenase-1; $\mathrm{I} / \mathrm{R}$, ischemia-reperfusion; ODQ, [1H-[1,2,4] Oxadiazole[4,3-a]quinoxalin-1-one]; PTIO, 2-phenyl-4,4,5,5-tetramethylimidazoline-1-oxyl-3-oxide; RSNO, mercury-labile NO-modified protein; RxNO, mercury-stable NO-modified protein; sGC, soluble guanylate cyclase; ZnDPBG, zinc (II) deuteroporphyrin IX 2,4-bis-ethylene glycol.

Conflict of interest: The NIH has submitted a provisional patent on the use of nitrite in ischemia-reperfusion. D.J. Lefer and M.T. Gladwin are listed as co-inventors.

Citation for this article: J. Clin. Invest. 115:1232-1240 (2005).

doi:10.1172/JCI200522493.
Although reperfusion of ischemic tissues provides oxygen and metabolic substrates necessary for the recovery and survival of reversibly injured cells, reperfusion itself actually results in the acceleration of cellular necrosis (15). I/R is characterized by the formation of oxygen radicals upon reintroduction of molecular oxygen to ischemic tissues, resulting in widespread lipid and protein oxidative modifications, mitochondrial injury, and tissue apoptosis and necrosis (16). In addition, after reperfusion of ischemic tissues, blood flow may not return uniformly to all portions of the ischemic tissues, a phenomenon that has been termed the "no-reflow" phenomenon (17). Reductions in blood flow after reperfusion are thought to contribute to cellular injury and necrosis (17). The sudden re-introduction of blood into ischemic tissue also results in massive tissue disruption, enzyme release, reductions in high energy phosphate stores, mitochondrial injury, and necrosis $(18,19)$. Furthermore, previous studies have also indicated that the I/R injury is characterized by an inappropriate inflammatory response in the microcirculation, resulting in leukocyteendothelial cell interactions that are mediated by the upregulation of both leukocyte and endothelial cell adhesion molecules $(20,21)$. Intensive research efforts have been focused on the amelioration of various pathophysiological components of $\mathrm{I} / \mathrm{R}$ injury to limit the extent of tissue injury and necrosis.

Nitric oxide, NO donors, and NO synthase activation or transgenic overexpression have been shown to exert protective effects on this process in a number of models (22-27) but in other models appear harmful (28-31). Evaluation of those studies suggests a critical effect of dose and duration of NO exposure, resulting in a 

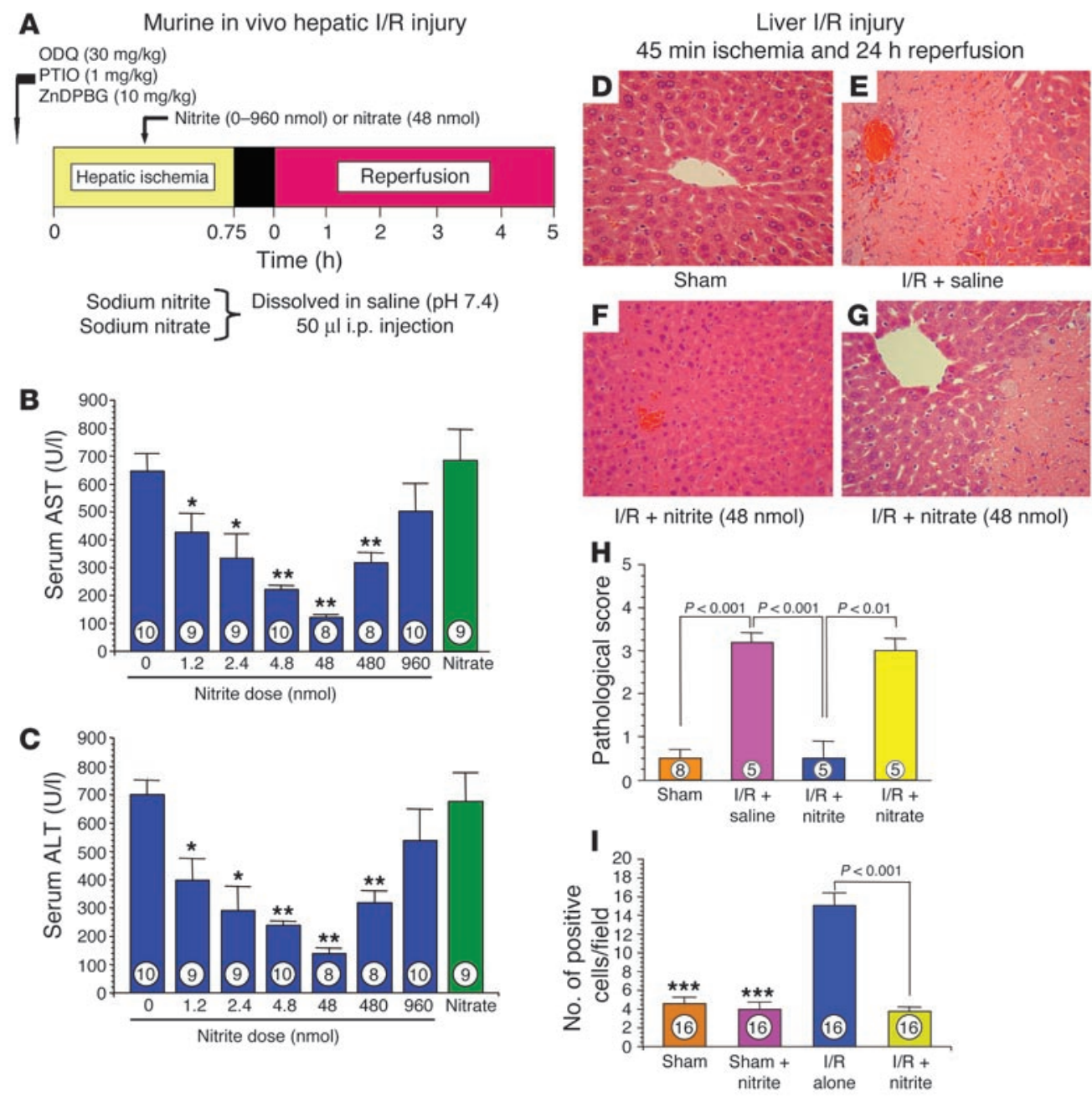

\section{Figure 1}

Nitrite therapy in hepatic I/R injury. (A) Experimental protocol for murine model of hepatic I/R injury. (B) Serum AST levels in mice after hepatic I/R. ${ }^{*} P<0.05$ vs. vehicle $(0 \mu \mathrm{M}) ;{ }^{* \star} P<0.01$ vs. vehicle $(0 \mu \mathrm{M})$. (C) Serum ALT levels in mice after hepatic I/R. (D-G) Representative photomicrographs of hepatic histopathology after 45 minutes of ischemia and 24 hours of reperfusion. Sham-treated (D) and l/R-injured mice treated with saline $(\mathbf{E})$, nitrite $(\mathbf{F})$, or nitrate $(\mathbf{G})$ are shown. $(\mathbf{H})$ Hepatic tissue sample pathology scores after 45 minutes of ischemia and 24 hours of reperfusion. (I) Hepatocellular apoptosis as measured by TUNEL staining after 45 minutes of ischemia and 24 hours of reperfusion. ${ }^{\star * \star} P<0.001$ vs. I/R alone. Numbers inside the bars indicate the number of mice investigated in each group. In D-I, $48 \mathrm{nmol}$ nitrate and nitrite were administered intraperitoneally.

narrow therapeutic safety window for $\mathrm{NO}$ in $\mathrm{I} / \mathrm{R}$ pathophysiology $(32,33)$. An additional limitation is that NO formation from NO synthase requires oxygen as substrate, a molecule whose availability becomes limited during ischemia. We therefore considered the use of nitrite in this context for the following reasons: (a) it is a naturally occurring substance with no potentially toxic "leaving group"; (b) it is selectively reduced to $\mathrm{NO}$ in tissues with low oxygen tension and low $\mathrm{pH}(4,6-13,34,35)$; and (c) $\mathrm{NO}$ is known to maintain heme proteins in a reduced and liganded state (36-38), to limit free iron- and heme-mediated oxidative chemistry (39-41), to transiently inhibit cytochrome c oxidase and mitochondrial respiration (42-45), and to modulate apoptotic effectors (46), all mechanisms that might participate in cytotoxicity after severe ischemia.

To test this hypothesis, we evaluated the effects of nitrite therapy compared with those of control therapy with vehicle or nitrate in well characterized in vivo murine models of hepatic and myocar- dial I/R injury. We provide strong evidence for a profound protective effect of low concentrations of nitrite on cellular necrosis and apoptosis, mediated by a hypoxia-dependent bioconversion of nitrite to NO and nitrosated or nitrosylated proteins.

\section{Results}

Intraperitoneal nitrite limits hepatic I/R injury. Intraperitoneal delivery of 1.2-960 nmol sodium nitrite during hepatic ischemia limited serum elevations of the liver transaminases aspartate aminotransferase (AST) and alanine aminotransferase (ALT) in a dose-dependent way (Figure 1, A-C), with a peak effect occurring at a dose of $48 \mathrm{nmol}$ nitrite. In sharp contrast, treatment with $0.9 \%$ saline or sodium nitrate $(48 \mathrm{nmol})$ did not exert any protective effects in the setting of hepatic I/R injury. Consistent with consumption of the intraperitoneal nitrite by the liver, plasma nitrite levels were not significantly elevated (baseline nitrite levels 
A Murine in vivo myocardial l/R protocol

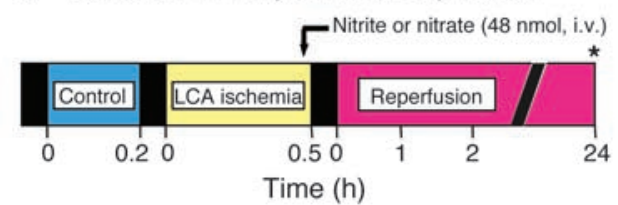

B Mouse myocardial infarction $30 \min \mathrm{Ml}+24 \mathrm{~h}$ reperfusion

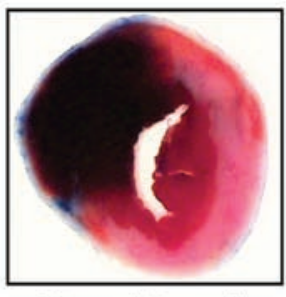

Nitrate $(48 \mathrm{nmol})$

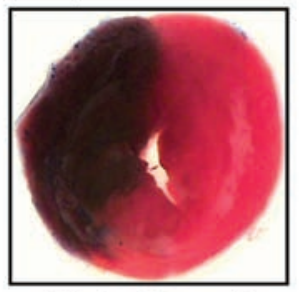

Nitrite $(48 \mathrm{nmol})$

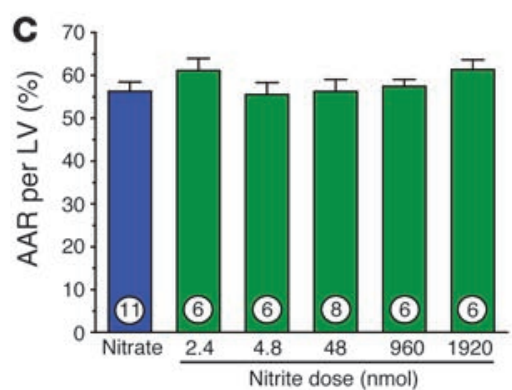

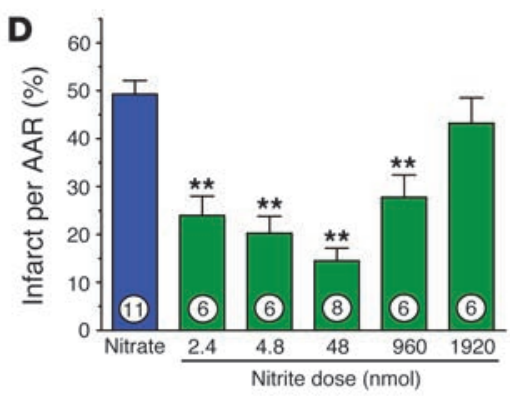

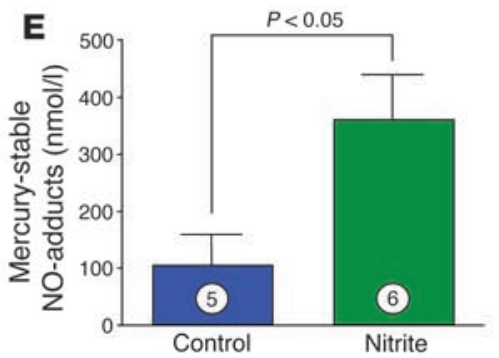

$\mathbf{F}$

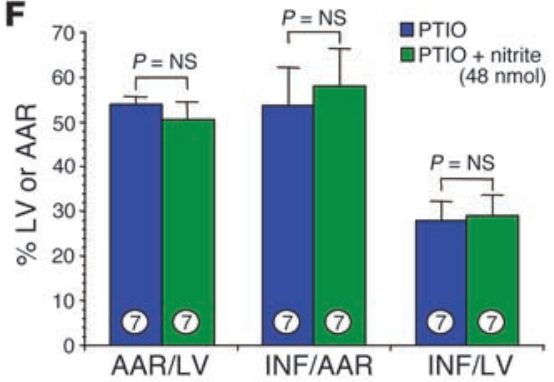

G

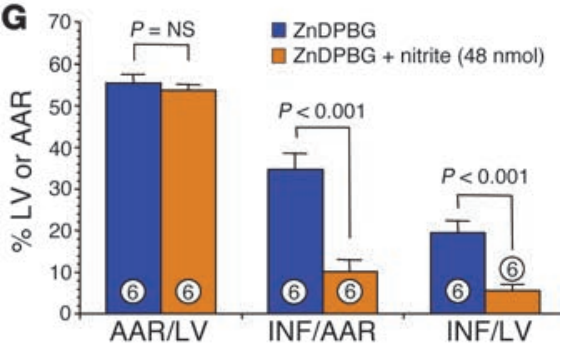

Figure 2

Nitrite therapy in myocardial I/R injury. (A) Experimental protocol for myocardial I/R studies in mice. Asterisk indicates in vivo AAR as shown by Evans blue injection, with infarct size $1.0 \%$ of 2,3,5-triphenyltetrazolium chloride (TTC). (B) Representative photomicrographs of murine hearts after 30 minutes of myocardial ischemia (MI) and 24 hours of reperfusion. Areas of the myocardium that appear blue (i.e., Evans blue dye) represent the areas of myocardium not at risk for infarction. In contrast, the areas of myocardium that stain red (i.e., TTC-positive) represent viable myocardium that was at risk for infarction. Myocardium that appears pale (i.e., TTC-negative) indicates areas of myocardium at risk that are necrotic (i.e., infarcted). Nitrite treatment significantly reduced myocardial infarction after 30 minutes of myocardial ischemia and 24 hours of reperfusion. (C) Myocardial AAR per LV for mouse hearts receiving nitrate (48 nmol) or doses of nitrite ranging from 2.4 to $1,920 \mathrm{nmol}$. The myocardial AAR per LV was similar for all of the study groups ( $P=$ NS between groups). (D) Myocardial infarct size per AAR for mouse hearts receiving nitrate $(48 \mathrm{nmol})$ or doses of nitrite ranging from 2.4 to $1,920 \mathrm{nmol}$. Nitrite therapy $(2.4-960 \mathrm{nmol})$ significantly reduced myocardial infarct size compared with nitrate therapy $\left.{ }^{* *} P<0.001\right)$. (E) Blood levels of iron-nitrosylated hemoglobin after intraventricular injection of $48 \mathrm{nmol}$ nitrite. (F) Myocardial AAR per LV and infarct size (INF) per AAR and per LV in mice treated with the NO scavenger PTIO prior to nitrite therapy (48 nmol). Nitrite-mediated cardioprotection was not observed in the presence of PTIO ( $P=\mathrm{NS}$, PTIO versus PTIO + nitrite). (G) Myocardial AAR per LV and INF per AAR and per LV in mice treated with the HO-1 inhibitor ZnDPGG prior to nitrite therapy (48 nmol). Treatment with ZnDPGG did not attenuate the protective effects of nitrite therapy. Numbers inside bars indicate the number of animals that were investigated in each group.

of $594 \pm 83 \mathrm{nM}$, which increased to $727 \pm 40 \mathrm{nM} ; n=3 ; P=0.16)$ in mice treated with $48 \mathrm{nmol}$ nitrite, the most effective dose.

Additional studies were performed for evaluation of the effects of nitrite treatment on hepatocellular injury in mice after in vivo hepatic ischemia (45 minutes) and more prolonged reperfusion (24 hours; Figure 1, D-I). The administration of $48 \mathrm{nmol}$ nitrite significantly reduced hepatocellular injury at 24 hours of reperfusion compared with the administration of saline or nitrate. In addition, nitrite therapy significantly attenuated the extent of hepatocellular apoptosis after 45 minutes of hepatic ischemia and 24 hours of reperfusion $(P<0.001$; Figure 1I). The extent of hepatic cell apoptosis in nitrite-treated animals subjected to I/R was similar to that observed in sham-operated control animals $(P=\mathrm{NS})$. Consistent with this treatment having a U-shaped efficacy profile, administration of $960 \mathrm{nmol}$ nitrite to mice subjected to hepatic I/R injury failed to exert a significant protective effect (also evidenced by the transaminase profile in Figure 1, B and C).
Intraventricular nitrite limits myocardial $I / R$ injury. To determine whether the potent cytoprotective effects of nitrite on liver $I / R$ injury could be generalized to other organ systems, we next performed studies to evaluate the potential cardioprotective effects of acute nitrite therapy in the setting of coronary artery occlusion and reperfusion. The experimental protocol for the myocardial I/R studies is depicted in Figure 2A. The administration of nitrite $(48 \mathrm{nmol})$ into the left ventricular cavity at 5 minutes prior to reperfusion significantly limited myocardial infarct size $(P<0.001$; Figure 2B) compared with the control treatment of 48 nmol nitrate. Despite the results showing similar myocardial area at risk (AAR) $(P=$ NS between groups; Figure $2 \mathrm{C})$, nitrate treatment decreased myocardial infarct size relative to the AAR and the left ventricle by $67 \%$ compared with nitrate-treated controls.

In additional groups of animals, we investigated the effects of various dosages of nitrite on myocardial infarct size after ischemia and reperfusion (Figure 2, C and D). Dosages of nitrite from 2.4 
A
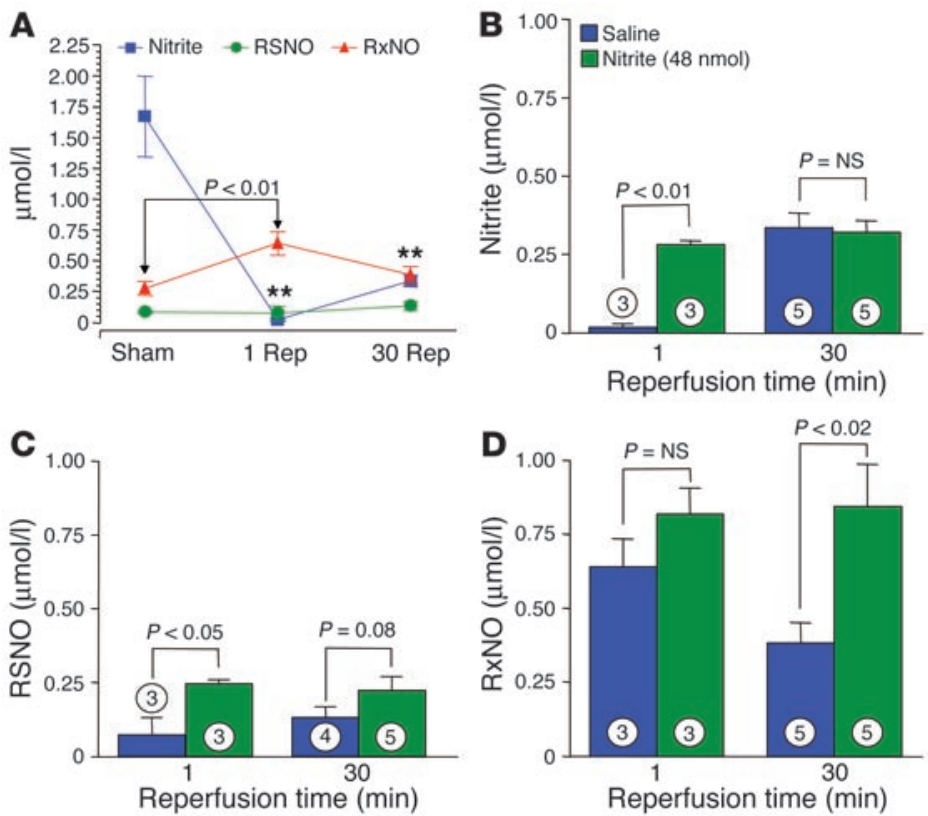

Figure 3

Blood and liver tissue levels of nitrite, RSNO, and RxNO. (A) Liver nitrite, RSNO, and RxNO levels $(\mu \mathrm{mol} / \mathrm{l})$ in animals ( $n=3-5$ per group) subjected to sham hepatic I/R (Sham) or to hepatic ischemia plus either 1 minute (1 Rep) or 30 minutes (30 Rep) of reperfusion. Experiments are saline-treated controls without nitrite. ${ }^{* \star} P<0.001$ vs. baseline. (B) Liver tissue nitrite levels in mice ( $n=3-5$ per group) subjected to hepatic I/R injury. (C) Liver tissue RSNO levels ( $\mu \mathrm{mol} / \mathrm{l})$ in mice $(n=3-5$ per group) subjected to hepatic ischemia and varying periods of reperfusion. (D) Hepatic tissue RxNO levels ( $\mu \mathrm{mol} / \mathrm{l})$ after hepatic ischemia and reperfusion in mice ( $n=3-5$ per group). Experiments in $\mathbf{B}-\mathbf{D}$ compare saline-treated controls to those with nitrite treatment $(48 \mathrm{nmol})$. Numbers inside bars indicate the number of mice investigated in each group.

treated control mice subjected to ischemia decreased from $1.75 \mu \mathrm{M}$ to undetectable $(P<0.001$ versus sham group) and levels of mercury-stable NO-modified proteins (representing $\mathrm{N}$-nitrosamines and iron-nitrosyl proteins and collectively referred to here as RxNOs) increased to approximately 750 $\mathrm{nM}(P<0.001$; Figure 3A). Interestingly, for nitrite treatment compared with saline treatment, there was a signifi-

to $1,920 \mathrm{nmol}$ were investigated. Data for myocardial AAR per left ventricle are presented in Figure 2C and clearly indicate that the AAR per left ventricle was similar in all study groups $(P=\mathrm{NS}$ between groups). We observed a dose-dependent decrease in myocardial infarct size with a maximal protective effect at $48 \mathrm{nmol}$ (Figure 2D). However, administration of $1,920 \mathrm{nmol}$ of nitrite failed to exert any significant cardioprotective effect.

To establish the plasma concentrations of nitrite that were cardioprotective, in additional experiments we injected increasing amounts of nitrite into the left ventricular chamber and sacrificed the animals and measured the concentration of nitrite in blood 5 minutes later. The plasma nitrite concentration correlated with the amount of nitrite injected $(\mu \mathrm{M}$ nitrite concentration in plasma, $0.965+0.1971 \times X+0.0006815 \times X^{2}$, where $X=$ the amount of nitrite injected in nanomoles; $n=16 ; r=0.96 ; P<0.001$ ). For the cardiac I/R experiments, the amounts injected and the measured concentrations in plasma were $0 \mathrm{nmol}$ and $0.965 \mu \mathrm{M}$ (basal plasma concentration of nitrite), $1.2 \mathrm{nmol}$ and $1.2 \mu \mathrm{M}, 2.4 \mathrm{nmol}$ and $1.4 \mu \mathrm{M}, 4.8 \mathrm{nmol}$ and $1.9 \mu \mathrm{M}, 48 \mathrm{nmol}$ and $11.9 \mu \mathrm{M}, 480 \mathrm{nmol}$ and $252.6 \mu \mathrm{M}$, and $960 \mathrm{nmol}$ and $818.3 \mu \mathrm{M}$. Consistent with the intravascular conversion of nitrite to $\mathrm{NO}(8)$, after the injection of $48 \mathrm{nmol}$ nitrite, a significant increase in iron-nitrosylated hemoglobin was observed (Figure 2E).

We performed additional studies of myocardial $\mathrm{I} / \mathrm{R}$ injury to determine possible mechanisms of nitrite-mediated cardioprotection (Figure 2, F and G). Pretreatment with the NO scavenger 2-phenyl-4,4,5,5-tetramethylimidazoline-1-oxyl-3-oxide (PTIO) completely abolished the protective effects of nitrite therapy (Figure 2F). In contrast, treatment with the heme oxygenase-1 (HO-1) inhibitor zinc (II) deuteroporphyrin IX 2,4-bis-ethylene glycol (ZnDPBG) did not attenuate the cardioprotective actions of nitrite therapy in the ischemic-reperfused myocardium (Figure 2G).

Nitrite-mediated cytoprotection is associated with the reduction of nitrite to $N O$ and formation of $S$-, $N$-, and heme-nitrosated or nitrosylated hepatic proteins. Consistent with previously described cellular reduction of nitrite to NO and S-nitrosothiols/N-nitrosamines $(4,8,9,34), 1$ minute after reperfusion the levels of nitrite in the livers of salinecant increase in liver levels of nitrite $(P<0.01$ versus saline; Figure 3B), S-nitrosothiols (Figure 3C), and RxNOs (Figure 3D) after reperfusion in the nitrite-treated mice.

These data suggest that nitrite is bioactivated during hypoxic stress and are consistent with our recent studies showing deoxyhemoglobin-dependent reduction of nitrite to $\mathrm{NO}(8)$, as well as recent studies by Bryan and colleagues demonstrating an acute conversion of tissue nitrite to mercury-labile NO-modified proteins (RSNOs) and RxNOs after a systemic anoxic insult (4). The low levels of nitrite that are cytoprotective $(1.2 \mathrm{nmol}$ at the lowest dose; Figure 1, B and C) and the observed reductive decomposition of "native" liver nitrite in the saline-treated control animals (Figure $3 \mathrm{~A}$ ) suggest that this may be an innate mechanism for hypoxic NO production and cytoprotection.

Cytoprotective effects of nitrite are NO dependent but independent of NO synthase and HO-1 enzymatic activities. Further supporting the idea of a mechanism involving the hypoxic reduction of nitrite to $\mathrm{NO}$, the NO inhibitor PTIO completely inhibited protective effects of nitrite in both heart (Figure 2F) and liver (Figure 4A). In contrast, nitrite remained cytoprotection in eNOS-deficient mice $(P<0.001$; Figure $4 \mathrm{~B}$ ), suggesting that $\mathrm{NO}$ production from nitrite during $\mathrm{I} / \mathrm{R}$ is eNOS independent. We investigated the role of soluble guanylate cyclase $(\mathrm{sGC})$ in nitrite-mediated protection against hepatic I/R injury diazole[4,3-a]quinoxalin-1-one] (ODQ) completely inhibited the protective effects of nitrite in the setting of hepatic $\mathrm{I} / \mathrm{R}$ injury.

Although HO- 1 mRNA and protein are both significantly induced after hepatic $\mathrm{I} / \mathrm{R}$ in this model, we did not observe any effects of nitrite treatment $(48 \mathrm{nmol}$ ) on HO-1 mRNA or protein levels during $\mathrm{I} / \mathrm{R}$ (Figure 4D). Furthermore, in mice pretreated with ZnDPBG, a specific and potent HO-1 inhibitor, nitrite significantly limited liver tissue injury, suggesting an $\mathrm{HO}-1$-independent effect $(P<0.05$; Figure $4 \mathrm{E})$, similar to experimental results during cardiac I/R (Figure 2G). Additional studies were performed utilizing HO-1-deficient $\left(H O-1^{--}\right)$mice that were treated with nitrite and subjected to hepatic I/R injury for further evaluation of the role of HO-1 in nitrite-mediated cytoprotection. We observed robust pro(Figure 4C). Pretreatment with the sGC inhibitor $[1 \mathrm{H}-[1,2,4]$ Oxa- 


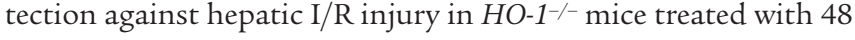
nmol nitrite $\left(P<0.01\right.$ versus the untreated $\mathrm{HO}-1^{-1-}$ group; Figure $4 \mathrm{~F})$, which further suggests that nitrite-induced protection does not involve HO-1 activation or signaling.

\section{Discussion}

In the present studies, nitrite treatment significantly increased the levels of liver nitrite and nitrosated or nitrosylated species (ironnitrosyl proteins, RSNO, and RXNO), compared with saline and nitrate control treatment, and conferred a substantial and unexpected dose-dependent cytoprotective effect, limiting necrosis and apoptosis and preserving organ function. Remarkably, a protective effect was observed with doses as low as $1.2 \mathrm{nmol}$ nitrite, suggesting that this may represent an endogenous protective mechanism that buffers severe metabolic or pathophysiological stress.

Recent data suggest that nitrite concentrations vary between blood and different organs and are typically in the high nanomolar to low micromolar range. However, until recently, the high concentrations required to vasodilate aortic ring preparations led to its dismissal as an important biologically active molecule. Indeed, Furchgott et al. demonstrated in 1953 that $100 \mu \mathrm{M}$ nitrite stimulated vasodilation of aortic ring preparations, a process later shown to be mediated by activation of sGC (47-50). From a physiological standpoint, the in vivo conversion of nitrite to $\mathrm{NO}$ was thought to be limited to the stomach and severely ischemic heart, where acidic reduction or disproportionation at very low $\mathrm{pH}$ produces gastric mucosal vasodilation $(5,11,51)$ and apparent cardiac tissue injury and heme iron-nitrosylation (at high nitrite concentrations in ischemic ex vivo heart preparations) (10), respectively. Although xanthine oxidoreductase-dependent nitrite reduction can occur at very low oxygen tensions and has recently been suggested to mediate nitrite-dependent cytoprotection in a Langendorff heart I/R model (52), NO production from this system is detectable only in the presence of high concentrations of superoxide dismutase $(53,54)$.

We recently reported that infusions of sodium nitrite into the human circulation produced significant vasodilation at both pharmacological and near-physiological concentrations (8). The bioactivation of nitrite appeared to be mediated by a nitrite reductase activity of deoxygenated hemoglobin, ultimately forming $\mathrm{NO}$ and iron-nitrosylated hemoglobin and, to a lesser extent, S-nitrosated protein species. Based on these data, a role for circulating nitrite in mediating hypoxic vasodilation was proposed, with the oxygen sensor in this case being hemoglobin (8). We hypothesize that a similar nitrite reductase activity of deoxyhemoglobin, deoxymyoglobin, and/or other deoxygenated heme proteins may account for the formation of nitrosated or nitrosylated proteins and apparent NO-dependent cytoprotection observed during liver and cardiac ischemia in our study here. The contribution of this mechanism versus pathways involving xanthine oxido-reductase and acidic reduction will require further study.

The precise mechanism of how nitrite confers tissue protection remains unclear, although a critical and direct role for $\mathrm{NO}$ is implied from data shown in our work here. Previous studies of $\mathrm{NO}$ and $\mathrm{I} / \mathrm{R}$ have yielded conflicting reports regarding the effects of $\mathrm{NO}$ on the severity of I/R injury, with some studies suggesting that $\mathrm{NO}$ actually contributed to reperfusion injury $(30,33)$. Our

\section{Figure 4}

Nitrite-mediated hepatoprotection and the $\mathrm{NO}$ and HO-1 signaling pathways. (A) Serum AST levels after hepatic I/R injury in mice receiving saline vehicle, nitrite (48 nmol), the NO scavenger PTIO, or nitrite (48 nmol) plus PTIO. ${ }^{* *} P<0.01$ vs. vehicle. (B) Serum levels of AST in eNOS-deficient $\left(e \mathrm{NOS}^{-/-}\right)$mice receiving saline vehicle or sodium nitrite $(48 \mathrm{nmol})$. (C) Serum levels of AST in mice receiving ODQ followed by nitrite (nitrite; $48 \mathrm{nmol}$ ) or the sGC inhibitor ODQ and in mice receiving ODQ pretreatment after nitrite (48 nmol; Nitrite + ODQ). (D) Hepatic tissue protein levels at 6 hours after ischemia determined using Western blot methods in animals subjected to sham and I/R with saline, nitrite, or nitrate. Hepatic tissue mRNA levels of HO-1 were determined using quantitative real-time RT-PCR methods in animals subjected to hepatic I/R. (E) Serum AST levels in mice treated with nitrite $(48 \mathrm{nmol})$ or the $\mathrm{HO}-1$ inhibitor ZnDPBG in the setting of hepatic l/R injury. (F) Serum AST and ALT levels in $\mathrm{HO}^{-1^{-/}}$mice treated with and without $48 \mathrm{nmol}$ of nitrite.
A

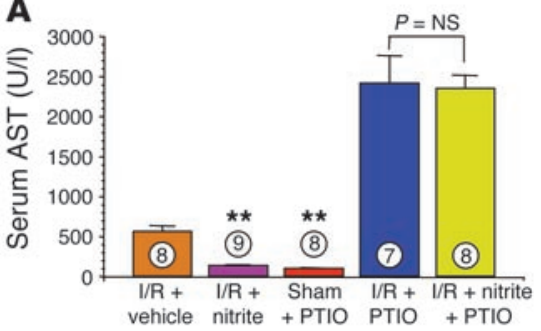

B
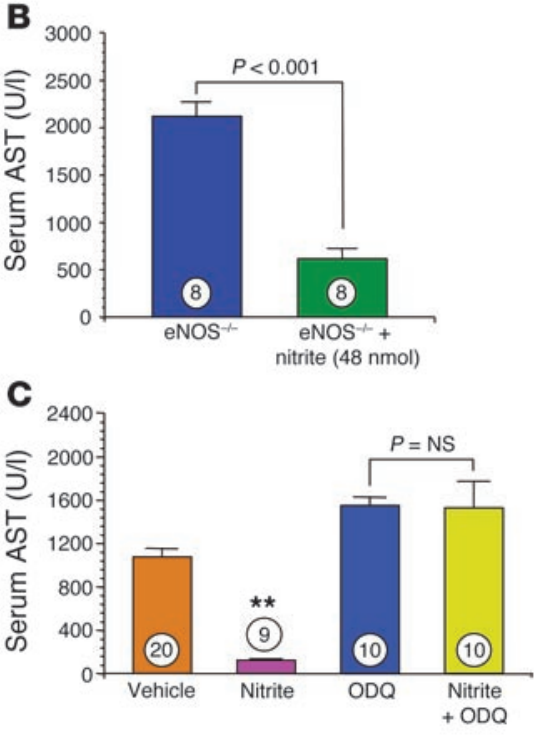

D
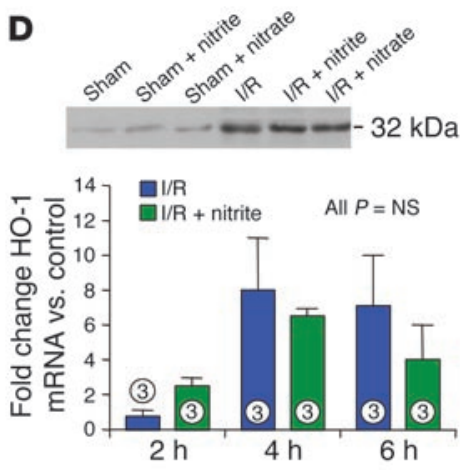

E
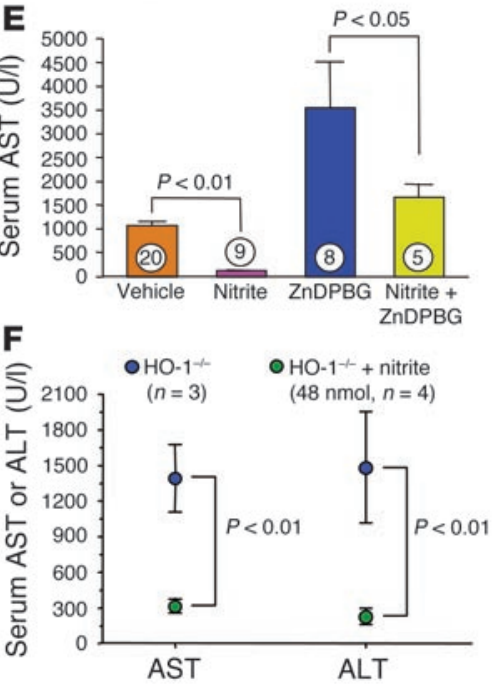
laboratory has previously demonstrated that NO donors as well as the NO precursor L-arginine protect against myocardial I/R injury $(22,24,55)$. More recently, we demonstrated that the severity of myocardial I/R injury is markedly exacerbated in mice completely deficient in eNOS (eNOS ${ }^{-/}$mice) (55), whereas mice with eNOS overexpression are protected against myocardial infarction and subsequent congestive heart failure $(25,26,56)$. Conflicting data on the effects of $\mathrm{NO}$ on I/R injury are probably related to the dose of $\mathrm{NO}$ and the conditions during ischemia and reperfusion (32). It is now well appreciated that very high, nonphysiological levels of $\mathrm{NO}$ (i.e., high micromolar and millimolar) actually promote cellular necrosis and apoptosis (57), while the demonstrated cytoprotective effects of NO typically involve nanomolar or low micromolar concentrations of $\mathrm{NO}(22,23,32)$. Additionally, studies investigating $\mathrm{NO}$ and NO-releasing agents under in vitro conditions of $\mathrm{I} / \mathrm{R}$ have consistently reported deleterious effects of NO (32), in contrast to in vivo studies of $\mathrm{I} / \mathrm{R}$ that have reported beneficial effects of NO therapy $(22,23)$. How NO mediates protection is also not clear, with multiple mechanisms being reported, including sGC activation, inhibition of cytochrome $\mathrm{C}$ oxidase, and inhibition of deleterious mitochondrial calcium uptake (42-45).

Although these data suggest that the effects of nitrite occur secondary to NO formation, the ultimate mechanism of nitritedependent cytoprotection is currently unknown. An intriguing possibility is the intermediate formation of S-nitrosothiols and $\mathrm{N}$-nitrosamines, known to form via reactions of nitrite with deoxyhemoglobin and possibly tissue heme proteins $(4,8,9,35,38,58$, 59). Consistent with hypoxia-dependent formation of S-nitrosothiols in red blood cells and tissues from nitrite, hepatic levels of these species were significantly higher after reperfusion (1-30 minutes) in livers exposed to ischemia and nitrite. Within the relative reductive environment intracellularly, S-nitrosothiols formed via nitrite will be readily reduced to $\mathrm{NO}$ and activate sGC. Alternatively, $\mathrm{S}$-nitrosation and subsequent effects on activity of critical proteins important in I/R-induced injury and apoptotic cell death may lead to protection (46). In addition, our data reveal a dynamic regulation of hepatic RxNOs, a pool of mercury-stable NO-modified proteins that include $\mathrm{N}$-nitrosamines and iron-nitrosyl proteins (4, $50,60)$, during I/R. In saline-treated groups, RxNO levels increased at 1 minute of reperfusion and then decreased after 30 minutes of reperfusion, whereas a sustained elevation in RxNO levels was observed in nitrite-treated mice, suggesting that the maintenance of RxNOs could be important in protecting tissues from I/R injury.

Finally, it is interesting that similar mechanisms might be at play in vivo and in isolated muscle during reductive meat-curing procedures. Nitrite has been utilized for curing meat for more than a century. During meat curing, the formation of iron-nitrosylated heme limits release of iron from the porphyrin molecule, prevents iron porphyrin- and metal ion-catalyzed lipid oxidation, Fenton chemistry, and ferryl heme formation (39-41, 61). Nitrite and NO produced from nitrite under hypoxic conditions can reduce ferryl hemoglobin $(36,37)$ and catalyze ferriheme protein reductive nitrosylation (38), and nitrite can react with deoxyhemoglobin to form NO, iron-nitrosyl-heme, and S- and N-nitrosated intermediates $(4,8,9,34,62)$. While it is clear that the formation of these reaction products in the mouse liver after $I / R$ are associated with cytoprotection, more work will be needed to determine the exact mechanisms of nitrite-induced cytoprotection.

In conclusion, these data demonstrate a remarkable function for the relatively simple inorganic anion nitrite as a potent inhibitor of liver and cardiac I/R injury and infarction in the mouse. The effects of nitrite appear to be NO dependent, with a rapid conversion of nitrite to $\mathrm{NO}$ and nitrosated or nitrosylated proteins after reperfusion. Given the known safety of nitrite as a naturally occurring anion and as an FDA-approved therapeutic for cyanide poisoning, these data evince a novel, safe, and inexpensive therapy for I/R injury. Such a therapy could be used to prevent or modulate organ dysfunction after coronary and peripheral vasculature reperfusion, high-risk abdominal surgery (such as aortic aneurism repair that leads to renal acute tubular necrosis), cardiopulmonary resuscitation, and, perhaps most importantly, solid organ transplantation. Further studies are required to establish the efficacy of nitrite in various organs and disease states.

\section{Methods}

Chemicals and reagents. Sodium nitrite (S-2252) and sodium nitrate (S-8170) were obtained from Sigma-Aldrich. Sodium nitrite and sodium nitrate were dissolved in phosphate-buffered saline and the $\mathrm{pH}$ was adjusted to 7.4. In all experiments, a final volume of $50 \mu \mathrm{l}$ containing $1.2-1,920 \mathrm{nmol}$ sodium nitrite or sodium nitrate was administered to the mice. Carboxy-PTIO [2-(4carboxyphenyl)-4,4,5,5-tetramethylimidazoline-1-oxyl-3-oxide potassium salt], a direct intravascular NO scavenger, was utilized to inhibit NO-dependent effects after both hepatic and myocardial I/R injury. Carboxy-PTIO (Alexis Biochemicals) was dissolved in phosphate-buffered saline and was administered intravenously at a dose of $1 \mathrm{mg} / \mathrm{kg}$ in a volume of $50 \mu \mathrm{l} 30$ minutes prior to either hepatic or myocardial ischemia. ZnDPBG (Alexis Biochemicals), an HO-1 inhibitor, was injected i.p. at a dose of $10 \mathrm{mg} / \mathrm{kg}$ in a volume of $50 \mu \mathrm{l} 30$ minutes prior to the induction of either hepatic or myocardial ischemia. $\mathrm{ZnDBG}$ has been shown previously to be a highly specific and potent inhibitor of HO-1 (63). The sGC inhibitor ODQ (Alexis Biochemicals) was utilized in experiments of hepatic I/R injury at a dose of $20 \mathrm{mg} / \mathrm{kg}$ in a volume of $50 \mu \mathrm{l} 30$ minutes prior to hepatic ischemia.

Animals. All of the mice utilized in the present studies were C57BL6/J at 8-10 weeks of age obtained from The Jackson Laboratory. In additional experiments of hepatic I/R injury we utilized $e \mathrm{NOS}^{-/-}$mice. The $e \mathrm{NOS}^{-/-}$ mice were originally generously donated by Paul Huang (Massachusetts General Hospital, Boston, Massachusetts, USA) and were generated in our breeding colony at Louisiana State University Health Sciences Center. The eNOS ${ }^{-/-}$mice were utilized at $8-10$ weeks of age. $\mathrm{HO}-1^{-/-}$mice were obtained from Shaw-Fang Yet (Harvard Medical School, Boston, Massachusetts, USA) and were utilized at 8-10 weeks of age.

Hepatic I/R protocol. The hepatic I/R protocol is depicted in Figure $1 \mathrm{~A}$ and has been described previously $(64,65)$. Mice were anesthetized with a combination of ketamine $(100 \mathrm{mg} / \mathrm{kg})$ and zylazine $(8 \mathrm{mg} / \mathrm{kg})$. Both ketamine and zylazine were injected intraperitoneally into the mice prior to all surgical procedures. A midline laparotomy incision was performed to expose the liver. Mice were then injected with heparin $(100 \mu \mathrm{g} / \mathrm{kg}$, i.p. $)$ to prevent blood clotting. The left lateral and median lobes of the liver were rendered ischemic by complete clamping of the hepatic artery and the portal vein using microaneurysm clamps. This experimental model results in a segmental (70\%) hepatic ischemia. This method of partial ischemia prevents mesenteric venous congestion by allowing portal decompression throughout the right and caudate lobes of the liver. The liver was then repositioned in the peritoneal cavity in its original location for 45 minutes. The liver was kept moist with gauze soaked in $0.9 \%$ normal saline. In addition, body temperature was maintained at $37^{\circ} \mathrm{C}$ using a heat lamp and monitoring of body temperature with a rectal temperature probe. Sham surgeries were identical except that hepatic blood flow was not reduced with a microaneurysm clamp. The duration of hepatic ischemia was 45 minutes in all experiments, after which the microaneurysm clamps were 
removed. The duration of hepatic reperfusion was 5 hours in the studies of serum liver transaminase levels (i.e., AST or ALT) and 24 hours for the studies of liver histopathology (i.e., hepatocellular infarction).

Liver enzyme determinations. Serum samples were analyzed for AST and ALT using a spectrophotometric method (Sigma-Aldrich) (66). These enzymes are liver specific and are released from the liver during injury $(64,65)$.

Liver histopathology studies. Histopathology of liver tissue was performed as reported previously (64). Liver tissue was fixed in $10 \%$ buffered formalin for 24 hours and was embedded in paraffin, and $10-\mu \mathrm{m}$ sections were stained with hematoxylin and eosin. Histopathology scoring was performed on randomly selected high-power fields by investigators blinded to sample identity using the following criteria: 0 , no hepatocellular damage; 1 , mild injury characterized by cytoplasmic vacuolization and focal nuclear pyknosis; 2 , moderate injury with dilated sinusoids, cytosolic vacuolization, and blurring of intercellular borders; 3 , moderate to severe injury with coagulative necrosis, abundant sinusoidal dilation, red blood cell extravasation into hepatic chords, and hypereosinophilia and margination of neutrophils; 4, severe necrosis with loss of hepatic architecture, disintegration of hepatic chords, hemorrhage, and neutrophil infiltration.

Hepatocellular apoptosis was determined using a TUNEL staining kit from Roche Diagnostics Corp. according to the manufacturer's recommendations. Briefly, liver tissue from various treatments was fixed in buffered formalin and $10-\mu \mathrm{m}$ sections were prepared. Sections were permeablilzed on ice for 2 minutes and were incubated in $50 \mu \mathrm{l} \mathrm{TUNEL}$ solution for 30 minutes at $37^{\circ} \mathrm{C}$. Sections were then treated with $50 \mu \mathrm{l}$ substrate solution for 10 minutes and were mounted under glass cover slips. The number of apoptotic nuclei was determined from 5 randomly selected $40 \times$ fields per specimen. A total of 6 specimens per treatment group (16 slides per group) were analyzed and compared using 1-way ANOVA with Bonferroni's post-testing.

Myocardial I/R protocol. Surgical ligation of the left main coronary artery was performed similar to methods described previously (25). Briefly, mice were anesthetized by intraperitoneal injection of ketamine $(50 \mathrm{mg} / \mathrm{kg})$ and pentobarbital sodium $(50 \mathrm{mg} / \mathrm{kg})$. The animals were then attached to a surgical board with their ventral side up. The mice were orally intubated with PE-90 polyethylene tubing connected to PE-240 tubing and then were connected to a Model 683 rodent ventilator (Harvard Apparatus). The tidal volume was set at 2.2 milliliters and the respiratory rate was set at 122 breaths per minute. The mice were supplemented with $100 \%$ oxygen via the ventilator side port. A median sternotomy was performed using an electric cautery and the proximal left main coronary artery was visualized and completely ligated with 7-0 silk suture mounted on a tapered needle (BV-1; Ethicon). In the initial experiments of myocardial infarct size, coronary occlusion was maintained for 30 minutes, followed by removal of the suture and reperfusion for 24 hours.

Myocardial infarct size determination. At 24 hours of reperfusion, the mice were anesthetized as described previously, intubated, and connected to a rodent ventilator. A catheter (PE-10 tubing) was placed in the common carotid artery to allow for Evans blue dye injection. A median sternotomy was performed and the left main coronary artery was re-ligated in the same location as before. Evans blue dye ( $1.2 \mathrm{ml}$ of a $2.0 \%$ solution; Sigma-Aldrich) was injected into the carotid artery catheter into the heart for delineation of the ischemic zone from the nonischemic zone. The heart was rapidly excised and serially sectioned along the short axis in 5 1-mm-thick sections that were then incubated in 1.0\% 2,3,5-triphenyltetrazolium chloride (Sigma-Aldrich) for 5 minutes at $37^{\circ} \mathrm{C}$ for demarcation of the viable and nonviable myocardium within the risk zone. Each of the 5 1-mm-thick myocardial slices were weighed and the areas of infarction, $\mathrm{AAR}$, and nonischemic left ventricle were assessed with computer-assisted planimetry (NIH Image 1.57) by an observer blinded to sample identity. All of the procedures for the left ventricular AAR and infarct size determination have been described previously (25).

HO-1 Western blot analysis. Liver tissue collected at 2,4, and 6 hours after $\mathrm{I} / \mathrm{R}$ injury was homogenized in radioimmunoprecipitation assay buffer with protease and phosphatase inhibitors and was centrifuged 3 times for 30 minutes at $4^{\circ} \mathrm{C}$; the supernatant was transferred to a fresh tube after each centrifugation. Protein concentrations were determined using BioRad reagents (BioRad Laboratories), and $50 \mu \mathrm{g}$ total protein from each sample was loaded. Western blots were performed using mouse $\mathrm{mAb}$ to HO-1 (Stressgen) at a 1:3,000 dilution and goat anti-mouse secondary HRP conjugate at a 1:2,000 dilution (Amersham Biosciences).

Blood and tissue nitrite and nitrosated or nitrosylated protein and hemoglobin determination. The concentration of nitrite in plasma and the levels of ironnitrosylated hemoglobin were measured using tri-idodide-based reductive chemiluminescence as described and validated previously $(67,68)$. Liver tissue was homogenized using an amended protocol published by Bryan and colleagues (4). Harvested liver tissue was blotted dry on filter paper, weighed, and homogenized immediately in ice-cold buffer containing $N$-ethylmaleimide (10 mmol/l)/diethylenetriaminepenta-acetic acid ( $2 \mathrm{mmol} / \mathrm{l})$ (3:1 dilution, $\mathrm{wt} / \mathrm{vol})$. The buffer/tissue mixture was then homogenized with a Wheaton glass-on-glass homogenizer. Tissue homogenates were kept on ice and were analyzed within 5 minutes. The homogenate was subsequently injected directly into tri-iodine for measurement of the sum of nitrite, RxNOs, and RSNOs. For determining the levels of specific NO adducts (RxNOs and RSNOs), the sample was treated with and without $5 \mathrm{mM}$ mercuric chloride (RSNO becomes nitrite in presence of mercuric chloride and $\mathrm{RxNO}$ is stable) and subsequently treated with acid sulfanilamide $(0.5 \%)$ to eliminate nitrite.

Real-time PCR protocol. Total RNA from liver tissue was isolated using the RNeasy kit (Qiagen Inc.) and the isolated RNA concentration was determined using a nanodrop ND-1000 spectrophotometer (Nanodrop Technologies). The quality and integrity of total RNA was assessed on an Agilent 2100 bioanalyzer. Quantitative real-time PCR assays for HO-1 and GAPDH transcripts were carried out using gene-specific double-fluorescence-labeled probes in a 7900 Sequence Detector (PE Applied Biosystems). Probes and primers for HO-1 and GAPDH were obtained from Applied Biosystems as Assays On Demand gene expression products.

In brief, first-strand cDNA was synthesized using $1 \mu \mathrm{g}$ of total RNA in a $20 \mu \mathrm{l}$ reverse transcriptase reaction mixture with Invitrogen Corp.'s Superscript cDNA synthesis kit, following the manufacturer's directions. PCR amplification was performed in a 384-well plate with a $20-\mu 1$ reaction mixture containing $300 \mathrm{~nm}$ of forward and reverse primers, $200 \mathrm{~nm}$ probe, $200 \mathrm{~nm}$ dNTP in $1 \times$ real-time PCR buffer, and passive reference (ROX) fluorochrome. The thermal cycling conditions were 2 minutes at $50^{\circ} \mathrm{C}$ and 10 minutes at $95^{\circ} \mathrm{C}$, followed by 40 cycles of 15 seconds of denaturation at $95^{\circ} \mathrm{C}$ and 1 minute of annealing and extension at $60^{\circ} \mathrm{C}$. The comparative threshold $\left(\mathrm{C}_{\mathrm{T}}\right) \mathrm{PCR}$ cycle detection method $\left(\Delta \Delta \mathrm{C}_{\mathrm{T}}\right.$ method) that compares the differences in $\mathrm{C}_{\mathrm{T}}$ values of control and treated groups was used to calculate the relative fold change in gene expression between groups.

Statistical analyses. Data were analyzed by 2 -way ANOVA with post-hoc Bonferroni analysis using StatView software version 5.0 (SAS Institute). Data are reported as mean \pm SEM. $P$ values less than 0.05 were considered significant.

\section{Acknowledgments}

These studies were supported by grants from the NIH (2RO1 HL60849 and PO-1 DK-43785, to D.J. Lefer) and by a grant from the American Diabetes Association (7-04-RA-59, to D.J. Lefer). These studies were also supported by the Intramural Research Division of the National Heart, Lung, and Blood Institute. 
Received for publication June 22, 2004, and accepted in revised form March 8, 2005.

Address correspondence to: David J. Lefer, Louisiana State University Health Sciences Center, 1501 Kings Highway, Shreveport, Louisiana 71130, USA. Phone: (318) 675-6974; Fax: (318) 675-
4217; E-mail: dlefer@lsuhsc.edu. Or to: Mark T. Gladwin, Building 10-CRC, Room 5-5140, 10 Center Drive; MS-1454, NIH, Bethesda, Maryland 20892-1454, USA. Phone: (301) 435-2310; Fax: (301) 402-1213; E-mail: mgladwin@nih.gov.

David J. Lefer and Mark T. Gladwin are co-senior authors.
1. Gladwin, M.T., et al. 2000. Role of circulating nitrite and S-nitrosohemoglobin in the regulation of regional blood flow in humans. Proc. Natl. Acad. Sci. U. S. A. 97:11482-11487.

2. Rodriguez, J., Maloney, R.E., Rassaf, T., Bryan, N.S., and Feelisch, M. 2003. Chemical nature of nitric oxide storage forms in rat vascular tissue. Proc. Natl. Acad. Sci. U. S. A. 100:336-341.

3. Rassaf, T., et al. 2003. NO adducts in mammalian red blood cells: too much or too little? Nat. Med. 9:481-483

4. Bryan, N.S., et al. 2004. Cellular targets and mechanisms of nitros(yl)ation: an insight into their nature and kinetics in vivo. Proc. Natl. Acad. Sci. U. S. A. 101:4308-4313.

5. Gladwin, M.T. 2004. Haldane, hot dogs, halitosis, and hypoxic vasodilation: the emerging biology of the nitrite anion. J. Clin. Invest. 113:19-21. doi:10.1172/JCI200420664.

6. Godber, B.L., et al. 2000. Reduction of nitrite to nitric oxide catalyzed by xanthine oxidoreductase. J. Biol. Chem. 275:7757-7763.

7. Li, H., Samouilov, A., Liu, X., and Zweier, J.L. 2001. Characterization of the magnitude and kinetics of xanthine oxidase-catalyzed nitrite reduction. Evaluation of its role in nitric oxide generation in anoxic tissues. J. Biol. Chem. 276:24482-24489.

8. Cosby, K., et al. 2003. Nitrite reduction to nitric oxide by deoxyhemoglobin vasodilates the human circulation. Nat. Med. 9:1498-1505.

9. Nagababu, E., Ramasamy, S., Abernethy, D.R., and Rifkind, J.M. 2003. Active nitric oxide produced in the red cell under hypoxic conditions by deoxyhemoglobin-mediated nitrite reduction. J. Biol. Chem. 278:46349-46356.

10. Tiravanti, E., Samouilov, A., and Zweier, J.L. 2004. Nitrosyl-heme complexes are formed in the ischemic heart: evidence of nitrite-derived nitric oxide formation, storage, and signaling in post-ischemic tissues. J. Biol. Chem. 279:11065-11073.

11. Zweier, J.L., Wang, P., Samouilov, A., and Kuppusamy, P. 1995. Enzyme-independent formation of nitric oxide in biological tissues. Nat. Med. 1:804-809.

12. Millar, T.M., et al. 1998. Xanthine oxidoreductase catalyses the reduction of nitrates and nitrite to nitric oxide under hypoxic conditions. FEBS Lett. 427:225-228.

13. Zhang, Z., et al. 1997. Human xanthine oxidase converts nitrite ions into nitric oxide (NO). Biochem. Soc. Trans. 25:524S.

14. Lauer, T., et al. 2001. Plasma nitrite rather than nitrate reflects regional endothelial nitric oxide synthase activity but lacks intrinsic vasodilator action. Proc. Natl. Acad. Sci. U. S. A. 98:12814-12819.

15. Braunwald, E., and Kloner, R.A. 1985. Myocardial reperfusion: A double-edged sword? J. Clin. Invest. 76:1713-1719.

16. McCord, J.M., Roy, R.S., and Schaffer, S.W. 1985. Free radicals and myocardial ischemia. The role of xanthine oxidase. Adv. Myocardiol. 5:183-189.

17. Kloner, R.A., Ganote, C.E., and Jennings, R.B. 1974. The "no-reflow" phenomenon after temporary coronary occlusion in the dog. J. Clin. Invest. 54:1496-1508.

18. Nayler, W.G. 1981. The role of calcium in the ischemic myocardium. Am. J. Pathol. 102:262-270.

19. Shen, A.C., and Jennings, R.B. 1972. Myocardial calcium and magnesium in acute ischemic injury.
Am. J. Pathol. 67:417-440.

20. Lefer, A.M., and Lefer, D.J. 1996. The role of nitric oxide and cell adhesion molecules on the microcirculation in ischaemia-reperfusion. Cardiovasc. Res. 32:743-751.

21. Entman, M.L., et al. 1991. Inflammation in the course of early myocardial ischemia. FASEB J. 5:2529-2537.

22. Lefer, D.J. 1995. Myocardial protective actions of nitric oxide donors after myocardial ischemia and reperfusion. New Horiz. 3:105-112.

23. Lefer, D.J., Nakanishi, K., Johnston, W.E., and Vinten-Johansen, J. 1993. Antineutrophil and myocardial protecting actions of a novel nitric oxide donor after acute myocardial ischemia and reperfusion of dogs. Circulation. 88:2337-2350.

24. Nakanishi, K., et al. 1992. Intracoronary L-arginine during reperfusion improves endothelial function and reduces infarct size. Am. J. Physiol. 263:H1650-H1658.

25. Jones, S.P., et al. 2004. Endothelial nitric oxide synthase overexpression attenuates myocardial reperfusion injury. Am. J. Physiol. Heart Circ. Physiol. 286: $\mathrm{H} 276-\mathrm{H} 282$.

26. Jones, S.P., et al. 2003. Endothelial nitric oxide synthase overexpression attenuates congestive heart failure in mice. Proc. Natl. Acad. Sci. U. S. A. 100:4891-4896.

27. Kanno, S., et al. 2000. Attenuation of myocardial ischemia/reperfusion injury by superinduction of inducible nitric oxide synthase. Circulation. 101:2742-2748.

28. Flogel, U., Decking, U.K., Godecke, A., and Schrader, J. 1999. Contribution of NO to ischemiareperfusion injury in the saline-perfused heart: a study in endothelial NO synthase knockout mice. J. Mol. Cell. Cardiol. 31:827-836.

29. Menezes, J., et al. 1999. A novel nitric oxide scavenger decreases liver injury and improves survival after hemorrhagic shock. Am. J. Physiol. 277:G144-G151.

30. Woolfson, R.G., Patel, V.C., Neild, G.H., and Yellon, D.M. 1995. Inhibition of nitric oxide synthesis reduces infarct size by an adenosine-dependent mechanism. Circulation. 91:1545-1551.

31. Schulz, R., and Wambolt, R. 1995. Inhibition of nitric oxide synthesis protects the isolated working rabbit heart from ischaemia-reperfusion injury. Cardiovasc. Res. 30:432-439.

32. Bolli, R. 2001. Cardioprotective function of inducible nitric oxide synthase and role of nitric oxide in myocardial ischemia and preconditioning: an overview of a decade of research. J. Mol. Cell Cardiol. 33:1897-1918.

33. Wink, D.A., et al. 2003. Orthogonal properties of the redox siblings nitroxyl and nitric oxide in the cardiovascular system: a novel redox paradigm. Am. J. Physiol. Heart Circ. Physiol. 285:H2264-H2276.

34. Doyle, M.P., Pickering, R.A., DeWeert, T.M., Hoekstra, J.W., and Pater, D. 1981. Kinetics and mechanism of the oxidation of human deoxyhemoglobin by nitrites. J. Biol. Chem. 256:12393-12398.

35. Luchsinger, B.P., et al. 2003. Routes to S-nitrosohemoglobin formation with heme redox and preferential reactivity in the beta subunits. Proc. Natl. Acad. Sci. U. S. A. 100:461-466.

36. Herold, S., and Rehmann, F.J. 2003. Kinetics of the reactions of nitrogen monoxide and nitrite with ferryl hemoglobin. Free Radic. Biol. Med. 34:531-545.

37. Herold, S., and Rehmann, F.J. 2001. Kinetic and mechanistic studies of the reactions of nitrogen monoxide and nitrite with ferryl myoglobin. J. Biol. Inorg. Chem. 6:543-555.

38. Fernandez, B.O., Lorkovic, I.M., and Ford, P.C. 2003. Nitrite catalyzes reductive nitrosylation of the water-soluble ferri-heme model FeIII(TPPS) to FeII(TPPS)(NO). Inorg. Chem. 42:2-4.

39. Kanner, J., and Harel, S. 1985. Initiation of membranal lipid peroxidation by activated metmyoglobin and methemoglobin. Arch. Biochem. Biophys. 237:314-321.

40. Kanner, J., and Harel, S. 1985. Lipid peroxidation and oxidation of several compounds by $\mathrm{H} 2 \mathrm{O} 2$ activated metmyoglobin. Lipids. 20:625-628.

41. Kanner, J., Harel, S., and Granit, R. 1992. Nitric oxide, an inhibitor of lipid oxidation by lipoxygenase, cyclooxygenase and hemoglobin. Lipids. 27:46-49.

42. Torres, J., Sharpe, M.A., Rosquist, A., Cooper, C.E., and Wilson, M.T. 2000. Cytochrome coxidase rapidly metabolises nitric oxide to nitrite. FEBS Lett. 475:263-266.

43. Brown, G.C., and Cooper, C.E. 1994. Nanomolar concentrations of nitric oxide reversibly inhibit synaptosomal respiration by competing with oxygen at cytochrome oxidase. FEBS Lett. 356:295-298.

44. Cleeter, M.W., Cooper, J.M., Darley-Usmar, V.M., Moncada, S., and Schapira, A.H. 1994. Reversible inhibition of cytochrome c oxidase, the terminal enzyme of the mitochondrial respiratory chain, by nitric oxide. Implications for neurodegenerative diseases. FEBS Lett. 345:50-54.

45. Rakhit, R.D., Mojet, M.H., Marber, M.S., and Duchen, M.R. 2001. Mitochondria as targets for nitric oxide-induced protection during simulated ischemia and reoxygenation in isolated neonatal cardiomyocytes. Circulation. 103:2617-2623.

46. Mannick, J.B., et al. 1999. Fas-induced caspase denitrosylation. Science. 284:651-654.

47. Kimura, H., Mittal, C.K., and Murad, F. 1975. Activation of guanylate cyclase from rat liver and other tissues by sodium azide. J. Biol. Chem. 250:8016-8022.

48. Mittal, C.K., Arnold, W.P., and Murad, F. 1978. Characterization of protein inhibitors of guanylate cyclase activation from rat heart and bovine lung. J. Biol. Chem. 253:1266-1271.

49. Ignarro, L.J., and Gruetter, C.A. 1980. Requirement of thiols for activation of coronary arterial guanylate cyclase by glyceryl trinitrate and sodium nitrite: possible involvement of S-nitrosothiols. Biochim. Biophys. Acta. 631:221-231.

50. Ignarro, L.J., et al. 1981. Mechanism of vascular smooth muscle relaxation by organic nitrates, nitrites, nitroprusside and nitric oxide: evidence for the involvement of S-nitrosothiols as active intermediates. J. Pharmacol. Exp. Ther. 218:739-749.

51. Bjorne, H.H., et al. 2004. Nitrite in saliva increases gastric mucosal blood flow and mucus thickness. J. Clin. Invest. 113:106-114. doi:10.1172/ JCI200419019.

52. Webb, A., et al. 2004. Reduction of nitrite to nitric oxide during ischemia protects against myocardial ischemia-reperfusion damage. Proc. Natl. Acad. Sci. U. S. A. 101:13683-13688.

53. Li, H., Samouilov, A., Liu, X., and Zweier, J.L. 2004. Characterization of the effects of oxygen on xanthine oxidase-mediated nitric oxide formation. J. Biol. Chem. 279:16939-16946.

54. Li, H., Samouilov, A., Liu, X., and Zweier, J.L. 2003. 
Characterization of the magnitude and kinetics of xanthine oxidase-catalyzed nitrate reduction: evaluation of its role in nitrite and nitric oxide generation in anoxic tissues. Biochemistry. 42:1150-1159.

55. Pabla, R., Buda, A.J., Flynn, D.M., Salzberg, D.B., and Lefer, D.J. 1995. Intracoronary nitric oxide improves postischemic coronary blood flow and myocardial contractile function. Am. J. Physiol. 269:H1113-H1121.

56. Jones, S.P., et al. 1999. Myocardial ischemiareperfusion injury is exacerbated in absence of endothelial cell nitric oxide synthase. Am. J. Physiol. 276:H1567-H1573.

57. Dimmeler, S., and Zeiher, A.M. 1997. Nitric oxide and apoptosis: another paradigm for the doubleedged role of nitric oxide. Nitric Oxide. 4:275-281.

58. Han, T.H., Hyduke, D.R., Vaughn, M.W., Fukuto, J.M., and Liao, J.C. 2002. Nitric oxide reaction with red blood cells and hemoglobin under heterogeneous conditions. Proc. Natl. Acad. Sci. U. S. A.
99:7763-7768.

59. Crawford, J.H., White, C.R., and Patel, R.P. 2003 Vasoactivity of S-nitrosohemoglobin: role of oxygen, heme, and NO oxidation states. Blood. 101:4408-4415.

60. Rassaf, T., Bryan, N.S., Kelm, M., and Feelisch, M. 2002. Concomitant presence of $\mathrm{N}$-nitroso and S-nitroso proteins in human plasma. Free Radic. Biol. Med. 33:1590-1596.

61. Pegg, R.B., and Shahidi, F. 2000. Nitrite Curing of Meat. Food \& Nutrition Press. Trumball, Connecticut, USA.

62. Herold, S. 2004. Nitrotyrosine, dityrosine, and nitrotryptophan formation from metmyoglobin, hydrogen peroxide, and nitrite. Free Radic. Biol. Med. 36:565-579.

63. Chernick, R.J., Martasek, P., Levere, R.D., Margreiter, R., and Abraham, N.G. 1989. Sensitivity of human tissue heme oxygenase to a new synthetic metalloporphyrin. Hepatology. 10:365-369.
64. Hines, I.N., et al. 2001. Enhanced post-ischemic liver injury in iNOS-deficient mice: a cautionary note. Biochem. Biophys. Res. Commun. 284:972-976.

65. Hines, I.N., et al. 2003. Regulation of postischemic liver injury following different durations of ischemia. Am. J. Physiol. Gastrointest. Liver Physiol. 284:G536-G545.

66. Harada, H., et al. 2003. Sexual dimorphism in reduced-size liver ischemia and reperfusion injury in mice: role of endothelial cell nitric oxide synthase. Proc. Natl. Acad. Sci. U. S. A. 100:739-744.

67. Gladwin, M.T., et al. 2002. S-nitrosohemoglobin is unstable in the reductive red cell environment and lacks O2/NO-linked allosteric function. J. Biol. Chem. 277:27818-27828.

68. Yang, B.K., Vivas, E.X., Reiter, C.D., and Gladwin, M.T. 2003. Methodologies for the sensitive and specific measurement of S-nitrosothiols, ironnitrosyls, and nitrite in biological samples. Free Radic. Res. 37:1-10. 